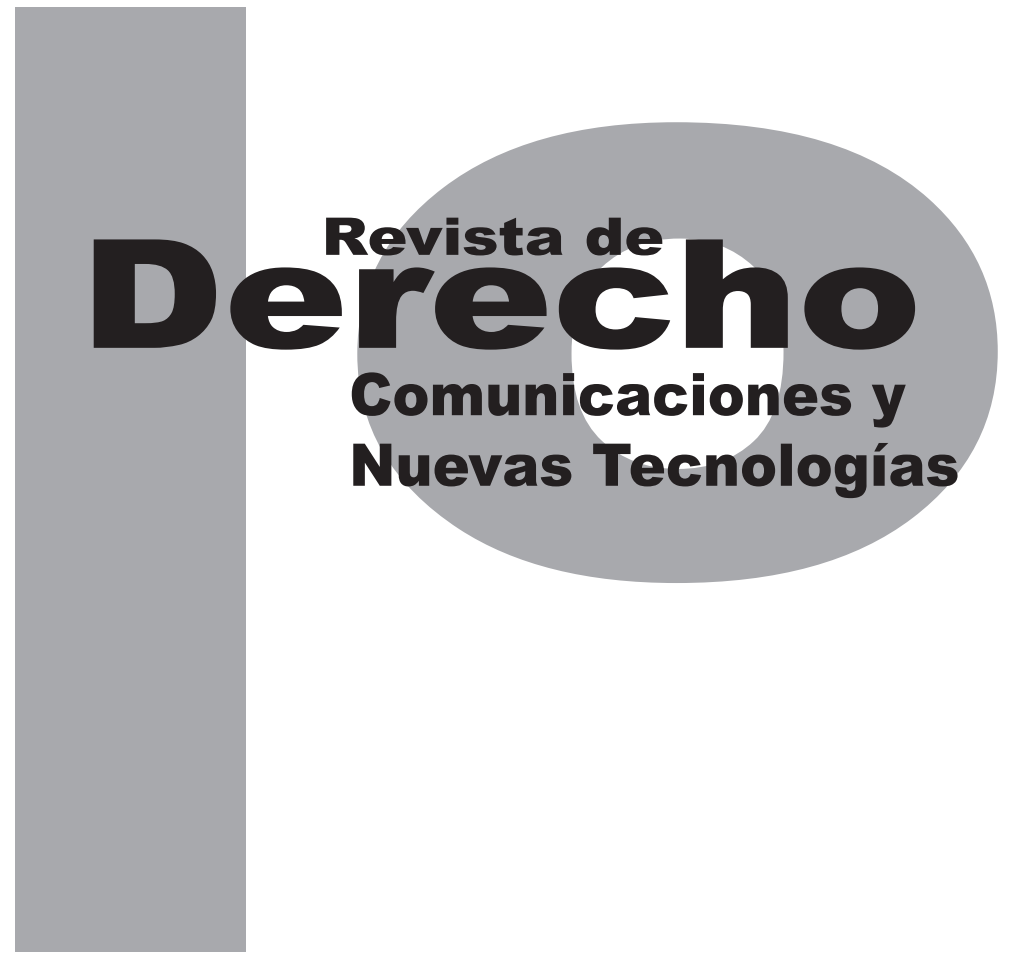

\title{
CONSENSO, CONFIANZA Y COOPERACIÓN EN EL DERECHO DEL ESPACIO ULTRATERRESTRE
}

\author{
ISABella María Franco Mogollón
}

Universidad de los Andes

Facultad de Derecho

Revista de Derecho, Comunicaciones y Nuevas Tecnologías

No.11, Enero - Junio de 2014. ISSN 1909-7786 


\title{
Consenso, confianza y cooperación en el derecho del espacio ultraterrestre ${ }^{1}$
}

\author{
Isabella María Franco Mogollón²
}

\section{RESUMEN}

Las normas del derecho del espacio ultraterrestre son de obligatorio cumplimiento. Esto no se debe únicamente al hecho de que hayan sido ratificadas por los Estados miembros, sino también por el hecho de que en este ámbito nos regimos por los principios de consenso, cooperación y confianza. Las normas del derecho del espacio ultraterrestre, como son los tratados, aunque no estén ratificados tienen que ser cumplidos debido a que todos los Estados miembros firmaron la Carta de las Naciones Unidas, la cual es considerada como fuente de derecho internacional y del derecho espacial. Adicionalmente, los principios, en virtud de la Convención de Viena, cuando no son ejercidos sobre ellos oposición persistente por parte de ningún Estado, se vuelven costumbre internacional, que es fuente de derecho internacional.
Compliance with the principles of outer space law is mandatory, not only because they were ratified by the Member States but also because we ought to abide by mutual cooperation, trust and consensus to be bound by them. Even though the principles of outer space law have not been ratified, such as treaties, ought to be complied with based on the member states' adoption of the United Nations Charter, which is considered the root of international law and outer space law. Additional principles, under the Viena Convention when it isn ' $t$ being exercised opposition persistency, become customary international law which is source of international law.

Cómo citar este artículo: Franco Mogollón, I. M. (Junio, 2014). Consenso, confianza y cooperación en el derecho del espacio ultraterrestre. Revista de Derecho, Comunicaciones y Nuevas Tecnologías, 11. 
Palabras clave: Derecho del espacio ultrate- Keywords: Outer space law, consensus, interrrestre, consenso, cooperación internacional, national cooperation, trust, treaties, principles. confianza, tratados, principios. 


\section{SUMARIO}

Introducción - I. LA HISTORIA DE LA NORMATIVIDAD DEL DERECHO DEL ESPACIO ULTRATERRESTRE - II. EL CONSENSO - A. Definición de consenso - B. Consenso en el derecho - C. Consenso en el derecho internacional - D. Consenso en el derecho del espacio ultraterrestre - III. LA CONFIANZA - A. Definición de confianza - B. Confianza en el derecho - C. Confianza en el derecho internacional - D. El concepto de confianza en el derecho del espacio ultraterrestre - IV. LA COOPERACIÓN INTERNACIONAL - A. Definición de la cooperación internacional - B. La cooperación internacional en el derecho internacional - C. Las convenciones de Viena - D. La cooperación internacional en el derecho del espacio ultraterrestre - V. NORMATIVIDAD DEL DERECHO DEL ESPACIO ULTRATERRESTRE APROBADA POR CONSESO - A. La Declaración de los principios jurídicos que deben regir las actividades de los Estados en la exploración y utilización del espacio ultraterrestre - B. Tratado sobre los principios que deben regir las actividades de los Estados en la exploración y utilización del espacio ultraterrestre, incluso la Luna y otros cuerpos celestes - C. Acuerdo sobre el salvamento y la devolución de astronautas y la restitución de objetos lanzados al espacio ultraterrestre - D. Convenio sobre la responsabilidad internacional por daños causados por objetos espaciales de 1972 - E. Convenio sobre el registro de objetos lanzados al espacio ultraterrestre de 1975 - F. Acuerdo que debe regir las actividades de los Estados en la Luna y otros cuerpos celestes de 1979 - VI. OBLIGATORIO CUMPLIMIENTO DE LAS NORMAS DEL DERECHO DEL ESPACIO ULTRATERRESTRE - VII. Conclusiones - Referencias. 
Introducción

El tema del derecho del espacio ultraterrestre ha sido generalmente desconocido debido a su novedad. Sin embargo, desde el lanzamiento del primer satélite artificial al espacio ultraterrestre ha aumentado el interés por el tema y cada día más personas se preguntan ¿qué es el espacio ultraterrestre?, ¿por qué es importante?, ¿cómo se regula?, ¿por qué se tiene que regular?, ¿se puede tener soberanía del espacio?

El derecho del espacio ultraterrestre tiene su origen en el conflicto internacional más importantes del siglo XX, la guerra fría entre la Unión Soviética y los Estados Unidos. Debido al enfrentamiento entre estas dos potencias mundiales, se empezó a desarrollar una fuerza armamentista debido a que se entendía que el espacio ultraterrestre era un nuevo territorio por conquistar, pero causaba un gran temor al resto de la comunidad internacional debido a que el hecho de colonizarlo y conquistarlo implicaría una militarización parecida, pero en mayor escala, a aquella experimentada en la Primera y la Segunda Guerra Mundial.

En consideración de la comunidad internacional, la Organización de las Naciones Unidas, por medio de la creación de la Comisión de Utilización del Espacio Ultraterrestre con Fines Pacíficos en 1959, junto con su Subcomisión Jurídica y su Subcomisión de Asuntos Científicos y Técnicos, empezó a buscar soluciones a este problema. Allí se da el origen de la normatividad del derecho del espacio ultraterrestre y la concertación de varios instrumentos jurídicos internacionales sobre los aspectos pacíficos de la utilización de dicho espacio.

Con el avance de los años, los Estados miembros de la Organización de las Naciones Unidas se han preocupado por la aplicación pacífica y la prevención de una posible militarización y de una carrera de armamentos en el espacio ultraterrestre. Paralelamente, también se han preocupado por el uso civil de las tecnologías espaciales.

Como consecuencia, en los últimos años ha aumentado el interés por fomentar la confianza mediante el aumento de un conjunto de normas encaminadas a aumentarla entre los Estados en general, y, en particular, en esferas concretas de sus actividades espaciales, y por medio de la aceptación de compromisos por parte de los Estados en materia de actividades espaciales.

\section{LA HISTORIA DE LA NORMATIVIDAD DEL DERECHO DEL ESPACIO ULTRATERRESTE}

El derecho del espacio es una rama del derecho internacional que se ha ido desarrollando a través de los avances tecnológicos y por medio de la progresiva conquista del espacio ultraterrestre, y tiene sus orígenes en uno de los conflictos internacionales más temidos: la guerra fría, en la cual eran protagonistas dos superpotencias, los Estados Unidos y la Unión Soviética. Hasta entonces solo había sido explotado el espacio aéreo, pero debido al enfrentamiento bélico que se estaba llevando a cabo entre las dos potencias mundiales, se empiezan a llevar a cabo los 
primeros desarrollos, tanto tecnológicos como legales. De los primeros que se llevó a cabo fueron los de las órbitas. Cuando Rusia mandó Sputnik 1 en 1957, comenzó la carrera espacial y con ella la preocupación por su regulación. Ese mismo año, los rusos enviaron el satélite Sputnik 2 con un ser vivo (la perra Laika). Así, 1957 fue el año del descubrimiento espacial y la carrera del espacio. ${ }^{3}$

Los Estados Unidos, preocupados por el uso que pudiera darle Rusia al espacio, comenzaron a buscar la forma de enviar un satélite al espacio lo antes posible. En 1958 se envió el Explorer 1 y el presidente Dwight D. Eisenhower creó la National Aeronautics and Space Administration (NASA), la cual opera como una agencia federal dedicada a la exploración espacial. En 1959, Rusia envió Luna 2, el primer objeto espacial en llegar a la Luna, y en 1961 enviaron a Yuri Gagarin, primer hombre en ser lanzado al espacio. En 1962, John Glenn se convirtió en el primer norteamericano en orbitar la Tierra. Finalmente, después de muchos intentos y avances tecnológicos, en 1968 los norteamericanos llegaron a tocar la Luna; Neil Armstrong y Buzz Aldrin fueron los primeros hombres en pisarla. Debido a la gran importancia de tener presencia física en el espacio ultraterrestre y el hecho de encontrarnos en medio de la guerra fría, se empezaron a desatar una serie de dudas: si la tecnología alcanzaba nuevas alturas, ¿la soberanía de los Estados debía existir también a esas alturas? ¿Se repetiría con los cuerpos celestes la experiencia

3 Sputnik and the Dawn of the Space Age, NASA. The Space Race. Recuperado de http://history.nasa.gov/sputnik/ colonial de las potencias europeas? ¿Los países de un bloque permitirían que un satélite del otro bando circulase libremente? ¿Se podrían almacenar armas nucleares en los satélites para dejarlos caer cuando estallase la tercera guerra?

Este debate se empezó a llevar a cabo en la ONU en ponencias del 17 de marzo de 1958, por parte de la Unión Soviética, y el 2 de septiembre del mismo año por parte de los Estados Unidos, en donde ambos Estados solicitaban que las actividades espaciales estuvieran cobijadas por el derecho internacional y la Carta de las Naciones Unidas. Adicionalmente, el resto de la comunidad internacional empezó a preocuparse por el hecho de que las superpotencias usaran el espacio ultraterrestre para expandir sus rivalidades militares, y que el espacio ultraterrestre quedara reservado para la explotación por un número limitado de países con los recursos necesarios, lo que provocó una pronta acción internacional para regularlo. Por esto, este mismo año se creó la Comisión de Utilización del Espacio Ultraterrestre con Fines Pacíficos (Copuos) con dos subcomisiones (la científica y técnica, y la jurídica), en las que se elaboraron las primeras normas que regulan el derecho espacial, utilizando el consenso como procedimiento para su aprobación. Igualmente, surgió el miedo por parte de los demás países de que las superpotencias usaran el espacio ultraterrestre para expandir sus rivalidades militares, o que este quedara reservado para la explotación por un número limitado de países con los recursos necesarios, lo que provocó una pronta acción internacional para regularlo. 
Posteriormente, en 1961, la Asamblea General de la ONU adoptó una nueva resolución, la 1721 (XVI) del 20 de diciembre de 1961, titulada Cooperación internacional para la utilización del espacio exterior con fines pacíficos, en la que se recalcó el interés de la humanidad por la utilización no violenta de esa nueva frontera. Con esta se realizaron los primeros esfuerzos de la extrapolación de los derechos ya utilizados en el uso terrestre, como aquellos relativos a los grandes pueblos y a sus constituciones, debido a que se había iniciado una "carrera espacial" que provocó el rápido desarrollo de las actividades espaciales que tuvieron en un principio fines fundamentalmente militares, y contaban con grandes cantidades de recursos económicos, financieros y tecnológicos. Por medio de esta resolución se buscó respetar aquellas leyes y normas que regulan las cuestiones generales del espacio como: a) no se autoriza la creación de un Estado o una colonia en un cuerpo celeste, ni la propiedad, pues solo se puede conceder el uso de la cosa por un tiempo determinado, y b) se debe tener la posibilidad de enviar muy rápidamente por satélites las noticias de uno a otro continente y de instruir a las sociedades aisladas geográfica y económicamente. Estas normas estaban dirigidas a respetar y favorecer la cooperación con vistas al bien común y a la paz en la Tierra y en el espacio. ${ }^{4}$ En 1963, la Asamblea General adoptó la Declaración de los principios jurídicos que gobiernan las actividades de los Estados en la exploración y utilización del espacio ultraterrestre, que sirvió de base para la elaboración

4 W. V. Rauchhaupt, H. El Sistema del Derecho Espacial, p. 203. Recuperado de http://www.ordenjuridico.gob.mx/Publicaciones/CDs2010/ CDEspacio/pdf/OP12.pdf del Tratado sobre los principios que deben regir las actividades de los Estados en la exploración y utilización del espacio ultraterrestre. ${ }^{5}$ El principio filosófico que influye permanentemente el derecho espacial es la buena fe, pacta sunt servanda, que implica que aunque los Estados no tengan siempre un fundamento normativo que los obligue a cumplir los mandatos internacionales, este principio es lo que los lleva a lograr lo pactado entre ellos, y de esta manera puede tener carácter obligatorio. "La confianza en las relaciones internacionales se basa en la fe, en la voluntad de cooperar de otros Estados. La confianza aumentará en la medida en que la conducta de los Estados indique su voluntad de demostrar una actitud no agresiva y de cooperación". ${ }^{6}$ Es por esto que esta buena fe se puede llevar a cabo mediante compromisos políticos o consensos, los cuales, eventualmente, pueden convertirse en obligaciones con arreglo al derecho internacional, es decir, en tratados vinculantes.

Esta resolución fue la base para la elaboración del Tratado sobre los principios que deben regir las actividades de los Estados en la exploración y utilización del espacio ultraterrestre, incluso la Luna y otros cuerpos celestes, que fue aprobado en 1967 y que es comúnmente conocido como el Tratado del Espacio o la Carta Magna del Espacio, ya que en él se establecen los principios

5 Aunque no tenía la fuerza vinculante de un tratado internacional, se consideró que constituía la base de un futuro tratado jurídicamente vinculante.

6 APÉNDICE II. Directrices sobre tipos apropiados de medidas de fomento de la confianza y sobre la aplicación de tales medidas en los planos mundial o regional. Asamblea General de las Naciones Unidas, Resolución 39/63 del 12 de diciembre de 1984. 
fundamentales a aplicarse en las actividades llevadas a cabo en el espacio ultraterrestre. Actualmente cuenta con 102 ratificaciones y 27 firmas, lo cual es un número extremadamente importante si se tiene en cuenta que la mayoría de los 191 Estados miembros de la ONU tienen muy poco contacto con las actividades llevadas a cabo en el espacio ultraterrestre.

Como se puede observar, en un margen de apenas diez años la humanidad logró revolucionarse y comenzó a conquistar el espacio. Esto empezó con los satélites y las órbitas y, por lo tanto, es preciso estudiar su importancia y regulación. El derecho espacial, a pesar de nacer de la guerra, es un derecho inclusivo y pacífico que busca el beneficio de toda la humanidad sin distinción de raza, etnia, o lugar de nacimiento, para al final beneficiar a toda la humanidad, y no solamente a los países de tecnología más desarrollada.

En 1979 finalizó la tendencia de crear a gran velocidad la normatividad que regulaba las nuevas tecnologías aplicadas al espacio ultraterrestre. El Copuos ha mantenido el esfuerzo de producir resoluciones que van a la Asamblea General de la ONU, conteniendo conjuntos de principios que regulan algunas categorías especiales de actividades espaciales. Sin embargo, muchos juristas han catalogado estos principios como soft law, ${ }^{7}$ pero hay que tomar en cuenta que si han sido aprobados por consenso, son un punto de partida para negociaciones futuras de otros convenios, y se reconocen como reglas de derecho consuetudinario. Un gran ejemplo

$7 \quad$ Las normas soft law carecen de fuerza vinculante. de estos son los derechos humanos, dado que la Declaración Universal de Derechos Humanos, que no ha adoptado forma de tratado y, por lo tanto, carece de carácter vinculante, ha sido incluida en las constituciones y leyes internas de Ios Estados, y muchos pactos y tratados se fundamentan en los principios plasmados en ella.

\section{EL CONSENSO}

\section{A. Definición de consenso}

El consenso es definido por la Real Academia Española como "un acuerdo producido por consentimiento entre todos los miembros de un grupo o entre varios grupos". Es una decisión que se toma según el acuerdo de la mayoría, al mismo tiempo que se trata de minimizar el nivel de conflicto con la minoría, dado que no requiere que se dé el consentimiento activo de todos los involucrados, pues es suficiente que "quienes se oponen a la decisión, atenúen su rechazo y toleren la disidencia". ${ }^{8}$ De esta manera, una sociedad puede alcanzar la paz social y vivir conjuntamente de manera armónica, y facilita la democracia.

La falta de consenso es el disenso o disentimiento. Es "una discrepancia, divergencia o una diferencia de ideas, pensamientos y posiciones, es una falta de acuerdo entre las partes". ${ }^{9}$ Esta consiste en no ajustarse al parecer de los de-

8 Definición de "consenso". Definición.De. Recuperado de http://definicion.de/consenso/

9 Ortiz, E. y Jesús M. Consenso y disenso. Recuperado de www.scribd. com/doc/83020100/Consenso-y-Disenso 
más, es decir que exista una oposición activa. Esta oposición no implica que exista un enfrentamiento, sino que es una posición que se asume para poder producir cambios en el consenso. Para que exista el consenso es necesario que se reúnan cinco elementos esenciales para poder invocarlo: 1) La voluntad para compartir el poder, 2) El compromiso consciente e informado con el proceso de consenso, 3) Una intención común, 4) Las agendas sólidas y 5) Una facilitación efectiva. ${ }^{10}$

En cuanto a la voluntad para compartir el poder es necesario que "las personas que participan en un grupo de consenso deban estar dispuestas a dejar ir las jerarquías y los privilegios para funcionar como iguales". ${ }^{11}$ Para que se dé el compromiso consciente e informado con el proceso de consenso es necesario que el grupo tome la decisión de usar el consenso. La intención común depende de "una misión abarcadora que unifique y enfoque sus esfuerzos", ${ }^{12}$ la cual puede ir cambiando con el tiempo, pero todo el grupo debe saber qué es lo que está cambiando y por qué se necesita el cambio. Una agenda sólida es necesaria y se lleva a cabo cuando el grupo "designa a unas cuantas personas a preparar la agenda y que, posteriormente, de manera colectiva, revisa la propuesta de agenda, la considera necesaria y la adopta formalmente

10 Briggs, B. Introducción al proceso de consenso. Instituto Internacional de Facilitación y Consenso, IIFAC, p. 6. Recuperado de http://proyectocultivandovida.pbworks.com/f/Introduccion+al+proceso+de+consen so.pdf

$11 \quad$ Ibid., p. 7.

12 Ibid. por consenso y se compromete a respetarla". ${ }^{13}$ Por último, la facilitación efectiva se lleva a cabo cuando existe un facilitador y este "es el guardián del proceso de consenso, es un dirigenteservidor cuya intención es ayudar al grupo a tomar las mejores decisiones posibles". ${ }^{4}$

La forma para llegar al consenso no es por medio del voto, sino por el consentimiento de la toma de una decisión, lo que implica que

todos los miembros de un grupo dicen que sí a una propuesta. Dar el consentimiento a una propuesta no implica necesariamente que a uno le encante cada aspecto de la propuesta, pero sí implica que, a pesar de los desacuerdos, uno está dispuesto a apoyar la decisión y permanecer solidario con el grupo. ${ }^{15}$

Además del consentimiento, los miembros de un grupo pueden tomar otras decisiones: bloquear o apartarse. Se puede bloquear cuando se "evita que se siga avanzando con la toma de una decisión, por lo menos por un tiempo". ${ }^{16}$ Es una decisión muy radical por tomar y solo se lleva a cabo cuando se cree que dar el consentimiento a una decisión implicaría poner en riesgo los valores, la seguridad y la misión del grupo en su totalidad. ${ }^{17}$ Es viable apartarse "cuando a nivel personal no puede o no quiere apoyar una propuesta, pero siente que estaría bien que el

13 Ibid.
14 Ibid., p. 8.
$15 \quad$ Ibid.
$16 \quad$ Ibid.
$17 \quad$ Ibid.


resto del grupo decida, aceptando cabalmente el resultado." 18

\section{B. Consenso en el derecho}

Es importante establecer que el derecho no es un sistema neutro sino que incluye diferentes puntos de vista: uno sobre lo moral, uno sobre la justicia y uno sobre la moralidad. Se caracteriza por poseer una innata vocación de eficacia y es el resultado de mecanismos sociales de producción. ${ }^{19}$ El consenso, en relación con un determinado ordenamiento jurídico, puede ser el fruto de la participación colectiva, puesto que "si bien la aceptación de un ordenamiento puede ser el resultado del acuerdo con el contenido de la norma, la participación contribuye a sentar las bases de la aceptación y, por lo tanto, de manera derivada, de la eficacia del sistema". ${ }^{20}$ El consenso adquiere "sentido en el marco de contextos participativos y deliberativos, más allá de los cuales el derecho es pura imposición". ${ }^{21}$ Sin embargo,

no es necesario para la creación de un sistema jurídico, no lo es para la creación de sus normas, para la toma de decisiones en su interior. El carácter necesario del consenso estará más o menos justificado en función de la materia en

$18 \quad$ Ibid.

19 Ansuátegui, F. J. Consenso y derecho, p. 59. Recuperado de http://earchivo.uc3m.es/bitstream/handle/10016/9399/consenso_ansuategui_2008.pdf;jsessionid=39DF33AAC578729435C6C0BC492DE506? sequence $=1$

20 Briggs, op. cit., p. 61

$21 \quad$ Ibid la que estamos pensando [...] [pues] los casos en los que se adoptan decisiones jurídicas sin consenso no son anormales, incluso en contextos democráticos. ${ }^{22}$

El consenso se puede evidenciar en la "configuración" de las constituciones de un sistema jurídico democrático como "compromisos en relación con determinados procedimientos y también en relación con determinados contenidos", y en la justificación de la limitación de la libertad, que son consecuencias necesarias del carácter normativo del derecho.

La naturaleza normativa del derecho implica una necesaria restricción en la libertad de los sujetos cuyos comportamientos son regulados por las normas jurídicas [...]. Esa restricción debe hacerse convivir con el principio general de la libertad, mediante el establecimiento de un criterio que establezca que las restricciones sean las mínimas y que estén justificadas. ${ }^{23}$

\section{Consenso en el derecho internacional}

El derecho internacional nace de los acuerdos creados entre miembros de la comunidad internacional (Estados). Estos pueden ser acuerdos multilaterales y/o bilaterales, y el valor del consentimiento individual de los Estados juega un papel importante dado que estos pueden quedar obligados, sin que lo digan expresamente, ya que el Estado se puede abstener de dar un sí explícito.

\footnotetext{
22 Ibid., p. 60.

23 Ibid., p. 67.
} 
Esto se debe a uno de los principios filosóficos fundamentales del derecho internacional, el pacta sunt servanda, el cual se entiende dentro de la Carta de la ONU como el hecho de que "Ios miembros de la Organización, a fin de asegurarse los derechos y beneficios inherentes a su condición de tales, cumplirán de buena fe las obligaciones contraídas por ellos de conformidad con esta Carta". Esto implica que aunque los Estados no se encuentren siempre obligados por medio de un fundamento normativo, por medio de la buena fe es como se logra llegar a consensos sobre diferentes temas.

Por medio de esta herramienta se logra, a través de las relaciones internacionales, crear un orden mundial más estable y duradero, garantizando la paz, pues cuando "Ios Estados toman decisiones por consenso implícitamente están reconociendo la necesidad de llegar a un acuerdo respecto de un asunto en específico, es decir, surge entre ellos una conciencia colectiva de luchar por una meta común". ${ }^{24}$ El consenso no es un acuerdo entre todos, puede simplemente ser un "no oponerse".

\section{Consenso en el derecho del espacio ultraterrestre}

Principios universales del derecho del espacio ultraterrestre, como lo son el principio del Uso Pacífico, el principio de Cooperación Internacional y el principio de No Apropiación, se encuen-

24 Varela, V. (2012). La importancia de fomentar la cooperación internacional en la utilización y exploración del espacio ultraterrestre (tesis de pregrado). Universidad Javeriana de Cali, Cali, Colombia. tran establecidos en el Tratado de 1967. Son normas que

se imponen a todos los sujetos de manera obligatoria, se basan en el consenso universal, sobre determinados valores mínimos, elementales, consideraciones de humanidad, intereses generales de la humanidad, que todos los Estados tienen que respetar al margen de toda voluntad expresa. Es así por la especial naturaleza del objeto jurídico que esta norma pretende proteger. Tienen alcance erga omnes (frente a todos). Se tratan de normas jurídicas indispensables para la vida de la comunidad internacional. ${ }^{25}$

Estos principios nacieron del compromiso político, o consenso, entre las potencias mundiales del siglo XX, la Unión Soviética y los Estados Unidos. Esto se debe a que en principio, la Unión Soviética buscaba gozar de un derecho absoIuto de veto en el Comité sobre la Utilización del Espacio Ultraterrestre con Fines Pacíficos, mientras que los Estados Unidos pretendían que todos se rigieran bajo el sistema de votos. Llegaron al compromiso de que las decisiones en este Comité se iban a tomar por medio de consenso, y que en medidas extremas se utilizaría el sistema de votos. Hoy en día, los cinco tratados existentes y cuatro de las cinco Declaraciones de Principios se han adoptado por consenso en el Comité. ${ }^{26}$

25

Principios que rigen a los tratados internacionales de derecho público. Universidad Sergio Arboleda. Recuperado de http://www.usergioarboleda.edu.co/derecho/derecho_constitucional/articulos_opinion_analisis_principiosquerigenlostratadosinternacionales.htm

26 Gutiérrez, C. La crisis del derecho del espacio. Un desafío para el derecho internacional del nuevo siglo, p. 249. Recuperado de http://dspace. unav.es/dspace/bitstream/10171/22202/1/ADI_XV_1999_06.pdf 
Aunque no exista un tratado ni un acuerdo vinculante que plasme cuál es la delimitación del espacio ultraterrestre, se entiende que la comunidad internacional, por medio del consenso, ha decidido (relativamente) que esta se da alrededor de los cien kilómetros de la Tierra. Sobre este punto, no ha habido una oposición persistente, que eliminaría dicho consenso. Esto, de manera conveniente, sirve para prevenir conflictos sobre la soberanía ya que al establecer límites establece la imposibilidad de la apropiación del espacio ultraterrestre por parte de los Estados. Este consenso nunca ha sido absoluto, y por lo tanto los Subcomités del Comité del Espacio solo se han limitado a emitir distintas teorías en cuanto a la delimitación. Esto se debe a que a este tema no se le puede imponer una definición, sino que se tiene que llegar a este por medio del consenso. Hoy en día es aceptada tácitamente la teoría de que el límite entre el espacio aéreo y el espacio ultraterrestre es de cien kilómetros, aunque no se encuentre plasmada en ninguna normatividad internacional.

\section{LA CONFIANZA}

\section{A. Definición de confianza}

La confianza, según Niklas Luhmann, es un "mecanismo de reducción de la complejidad y aumenta la tolerancia a la incertidumbre", ${ }^{27}$ y como lo describe Yolanda Onghena, "es una apuesta, hecha en el presente, hacia el futuro y

27 Onghena, Y. (2003). ¿Por qué la confianza? Revista CIDOB d'Afers Internacionals, (61-62), 9. que se fundamenta en el pasado". ${ }^{28}$ La base de la confianza es el presente y se basa en el hecho de que otros también confían y que esta posesión común de la confianza se hace consciente. Según Edgar Weber, es "un proceso que sale de lo particular y se interesa por lo universal, como un horizonte que se aleja a medida que nos acercamos y como un proceso construido con el Otro". ${ }^{29}$ Doctrinantes como Francis Fukuyama la define como "[...] la expectativa que surge dentro de una comunidad de comportamiento normal, honesto y cooperativo, basada en normas comunes, compartidas por todos los miembros de dicha comunidad", ${ }^{30}$ para poder ubicarlo como el elemento central que explica las diferencias en el origen y el desarrollo entre las naciones. Las normas comunes a las que se refiere son definidas como "capital social", los cuales son "el conjunto de valores o normas informales compartidas entre los miembros de un grupo, que permiten la cooperación entre los mismos", ${ }^{31}$ el cual es considerado como el elemento central para el desarrollo exitoso de cualquier sociedad, y lo que permite que funcionen es "la capacidad de confianza que pueden generar sus miembros

$28 \quad$ Ibid

29 Ibid., p.16.

30 Hevia de la Jara, F. ¿Cómo construir confianza? Hacia una definición relacional de la confianza social. Instituto de Investigaciones Jurídicas UNAM, p. 18. Recuperado de http://www.redacademica.edu.co/archivos/redacademica/proyectos/ddhh/autoformacion_ddhh/unidad10/ anexo_10-23_como_construir_confianza.pdf

31 Fukuyama, F. La gran ruptura. En: Hevia de la Jara, F. ¿Cómo construir confianza? Hacia una definición relacional de la confianza social. Instituto de Investigaciones Jurídicas UNAM, p. 18. Recuperado de http://www.redacademica.edu.co/archivos/redacademica/proyectos/ ddhh/autoformacion_ddhh/unidad10/anexo_10-23_como_construir_ confianza.pdf 
[...] [dado que] a mayor confianza, mayor capital social y mayor desarrollo". ${ }^{32}$

Por otro lado, la desconfianza "consiste en el hecho de que el futuro contiene muchas más posibilidades de las que podrían actualizarse en el presente, y del presente transferirse al pasado, lo que provoca incertidumbre" ${ }^{33}$ Es un aspecto central en las sociedades complejas y un concepto que proviene del "mundo del sentido común", ya que es un "concepto que se utiliza cotidianamente en nuestras conversaciones, donde asumen una capacidad explicativa por sí misma". ${ }^{34}$ Culturalmente la desconfianza es entendida como "la negación del otro" dado que "el 'yo' siempre buscará e inquirirá cualquier argumento para que aparezca el otro, pues lo necesita para mantener su dominación". ${ }^{35}$

\section{B. Confianza en el derecho}

La buena fe es un principio general del derecho y es reconocida como fuente del mismo. Esta se

desdobla en dos aspectos: primeramente cada persona debe usar para con aquel con quien establece una relación jurídica, una conducta sincera, vale decir, ajustada a las exigencias del decoro social; en segundo término, cada

32 Ibid.

33 Onghena, op. cit., p. 10.

34 Hevia de la Jara, op. cit., p. 16

35 Valero, J. Sociedades de cultura versus sociedades de desconfianza. Universidad de Valladolid, p. 213. Recuperado de http://www.google. com/url?sa=t\&rct=j\&q=\&esrc=s\&source=web\&cd=1\&ved=0CCgQFjA A\&url=http \%3A\%2F\%2Fdialnet.unirioja.es\%2Fdescarga\%2Farticulo \%2F1138368.pdf\&ei=TmOEUs7oH9CLkAeV54D4AQ\&usg=AFQjCNG 2wkMOxAxJkNmM3sPwlqn39e2_5A\&bvm=bv.56343320,d.ew0 persona tiene derecho a esperar de la otra la misma lealtad o fidelidad. En el primer caso se trata de una buena fe activa, y en el segundo, de una buena fe pasiva (confianza). ${ }^{36}$

Las actuaciones de confianza son actos jurídicos, como lo establece el doctrinante Ospina Fernández, que

deben ser cumplidos de buena fe, con entera lealtad, con intención recta y positiva, para que así pueda realizarse cabal y satisfactoriamente la finalidad social y privada a que obedece su celebración [...]. Tiene un alcance muy general, como quiera que es aplicable no solo a los contratos, sino a todos los actos jurídicos y, lo que es más, a todas las obligaciones, cualesquiera que sean fuentes. ${ }^{37}$

Entre las relaciones internacionales, "las medidas de fomento de confianza son aceptadas cada vez más como un elemento importante para reducir las sospechas y tensiones que existen entre las naciones y para afianzar la paz y la estabilidad internacional". ${ }^{38}$ Por medio de medidas bilaterales y multilaterales de fomento de la confianza se han evaluado las distintas maneras en que estas pueden ayudar a fortalecer las relaciones jurídicas entre los Estados.

Este proceso se lleva a cabo por medio de la reducción paulatina o completa eliminación de "causas de desconfianza, temor, malentendidos

\footnotetext{
36 Valencia Zea, A. (1981). Derecho Civil. Parte general y personas, t. I, 9. a ed., Bogotá: Temis, p. 196.

37 Ospina Fernández, G. (1998). Teoría general del contrato y del negocio jurídico, $5 .^{\text {a }}$ ed., Bogotá: Temis, p. 331.

$-$

38 Asamblea General de las Naciones Unidas, op. cit., p. 29.
} 
y errores de cálculo en lo que respecta a las capacidades militares $\mathrm{y} / \mathrm{o}$ de doble uso pertinentes de otros Estados así como de otras actividades suyas relativas a la seguridad". ${ }^{39}$ Esto recae en la premisa de que los Estados deben tener la satisfacción de estar enterados de cuáles son las actividades militares y de seguridad que están llevando a cabo los otros Estados, y que al mismo tiempo estas actividades no van a constituir una amenaza a su propia seguridad.

\section{Confianza en el derecho internacional}

La confianza en el derecho internacional se entiende como "la creencia de que otros Estados están dispuestos a cooperar, y de que esa confianza aumenta con el tiempo, a medida que la conducta de los Estados demuestra su disposición a comportarse de manera cooperativa", ${ }^{40}$ ya que por medio de estos se pueden reducir las sospechas y tensiones que existen entre los Estados. Esta se lleva a cabo a través de un proceso de fomentación que se entiende como

la eliminación de las causas de desconfianza, temor, malentendidos y errores de cálculo en lo que respecta a las actividades suyas relativas a la seguridad. Este proceso se basa en la premisa de reconocer que todo Estado necesita recibir garantías de que determinadas actividades militares o relativas a la seguridad realizadas por otros Estados no constituyen una amenaza para su propia seguridad. ${ }^{41}$

\footnotetext{
39 Ibid.

40 Asamblea General Naciones Unidas, op. cit., p. 30.

41 Ibid
}

Esta confianza se puede alcanzar cuando la cantidad de información a la que puede tener acceso un Estado le permitiría realizar las previsiones necesarias para poder calcular las acciones y reacciones de los demás Estados, ya que un gran nivel de previsibilidad aumenta la disposición para que se puedan dirigir los asuntos políticos y militares de manera transparente. ${ }^{42}$

Para que se apliquen medidas de fomento de la confianza es necesario que exista un consenso por parte de los Estados, y que este provenga del resultado de la voluntad política de los Estados en su libre ejercicio de la soberanía. ${ }^{43}$

Estas medidas de fomento de la confianza pueden ser aplicables a tres categorías de Estados: “a) los que participan directamente en actividades que pueden ser causa de desconfianza o tensión; b) otros Estados afectados por políticas militares o de seguridad de los Estados de la primera categoría, y c) los Estados cuya participación consiste en alentar el avance del proceso de fomento de la confianza". ${ }^{44}$

\section{El concepto de confianza en el derecho del espacio ultraterrestre}

La confianza en el derecho del espacio ultraterrestre se centra en la creación de medidas, por parte de los Estados, orientadas a reducir las preocupaciones y el temor de una futura existencia de ataques bélicos, que son creadas por
42 Ibid., p. 31.
43 Ibid., p. 32
44 Ibid., p. 33 
la presencia de actividades y por el desarrollo tecnológico espacial que existe. Este temor se debe a que las potencias espaciales tienen la capacidad de "penetrar en todos los puntos de la Tierra, para muy diversos fines civiles y militares. Esta capacidad de intrusión, incluso en los casos en que no entrañe armamentos, puede generar desconfianza". ${ }^{45}$ Por medio de la confianza se busca cumplir con el objetivo de generar seguridad en las actividades que son realizadas en el espacio ultraterrestre, especialmente en aquellas actividades militares como el armamentismo.

Como existen algunas actividades espaciales que se encuentran reguladas por acuerdos internacionales, tales como los procedimientos de publicación y notificación previas para todas las estaciones de satélites, también existe un gran número de actividades que no se encuentran reguladas por ningún acuerdo internacional concreto. La confianza entre los países se puede mejorar "mediante diversos sistemas capaces de vigilar las actividades militares terrestres", 46 ya que muchos sistemas tienen una función doble: civiles y militares. Esto se evidencia en el hecho de que la tecnología utilizada para el lanzamiento de satélites es similar a la utilizada para lanzar misiles balísticos de largo alcance. Los satélites utilizados para observar recursos naturales adicionalmente pueden obtener imágenes de interés para estrategias militares. ${ }^{47}$

\footnotetext{
45 Ibid., p. 36.

$46 \quad$ Ibid., p. 38.

47 Ibid. Los satélites de comunicaciones, meteorológicos, etc., tienen usos civiles y militares.
}

Sin embargo, por medio del cumplimiento de las cláusulas de notificaciones plasmadas en la Resolución 47/68 de la Asamblea General, relativas a los principios pertinentes a la utilización de fuentes de energía nuclear en el espacio ultraterrestre, se podría llegar a reducir la intranquilidad sobre el uso de dichos dispositivos en el espacio ultraterrestre, dado que por medio del suministro de más información sería una manera de demostrar la existencia de seguridad y control.

En los últimos años se han elaborado una serie de propuestas dirigidas a solucionar problemas a través de medidas concretas del fomento de la confianza, las cuales corresponden a las siguientes categorías:

a) las que tienen por objeto aumentar la transparencia de las operaciones espaciales en general; b) aquellas cuyo objetivo concreto es aumentar el alcance de la información sobre lo satélites en órbita; c) las encaminadas a establecer normas de comportamiento que rijan las operaciones espaciales; d) las relacionadas con la trasferencia internacional de tecnología espacial y de cohetes. ${ }^{48}$

Por medio de estas, se buscaría

reducir los peligros de incomprensión o error de cálculo en relación con las actividades militares, ayudar a prevenir un enfrentamiento militar, así como los preparativos encubiertos para iniciar una guerra, reducir el peligro de que se produzcan ataques por sorpresa y de que estalle la guerra accidentalmente; $y$, por úl-

$48 \quad$ Ibid., p. 46 
timo, dar cumplimiento y expresión concreta al compromiso solemne de todas las naciones de abstenerse de la amenaza o el uso de la fuerza en todas sus formas y fomentar la seguridad y la estabilidad. ${ }^{49}$

\section{LA COOPERACIÓN INTERNACIONAL}

\section{A. Definición de la cooperación internacional}

El término cooperación internacional se refiere al conjunto de acciones que derivan de los flujos de intercambio que se producen entre sociedades nacionales diferenciadas en la búsqueda de beneficios compartidos en los ámbitos del desarrollo económico y el bienestar social; o que se desprenden de las actividades que realizan tanto los organismos internacionales que integra la ONU como aquellos de carácter regional, intergubernamentales o no gubernamentales, en cumplimiento de intereses internacionales particularmente definidos. En términos más simple, se entiende como la movilización de recursos financieros, humanos, técnicos y tecnológicos para promover el desarrollo internacional.

Este concepto también salió a relucir con el inicio de conflictos internacionales que atemorizaron a la comunidad internacional: las dos guerras mundiales. Los primeros esfuerzos para consolidar la cooperación internacional se vieron con la creación de la ONU en 1945, dado que con la firma de las cincuenta naciones fundadoras se

49 Ibid., p. 98. reconoció a la cooperación internacional como un principio y práctica fundamental para la solución de los problemas de carácter económico, social, cultural, y para estimular el respeto de los derechos humanos y las libertades fundamentales. ${ }^{50}$

Hoy en día los Estados están sumergidos en un alto nivel de interdependencia entre ellos, lo cual ha generado el desarrollo de la cooperación internacional en diversos aspectos, en donde "los grandes desequilibrios económicos existentes entre las naciones del mundo han llevado a aquellos países económicamente más desarrollados a procurar ayuda a aquellos más atrasados. Las razones pueden encontrarse en motivos altruistas y morales o en otros más pragmáticos o interesados [...]". ${ }^{51}$ Debido a esto, se puede establecer que

la cooperación se produce cuando los actores adaptan sus conductas a las preferencias presentes o anticipadas de otros, por medio de un proceso de coordinación de políticas [...]. La cooperación intergubernamental se lleva a cabo cuando las políticas seguidas por un gobierno son consideradas por sus asociados como medio para facilitar la consecución de sus propios objetivos, como resultado de un proceso de coordinación de políticas. ${ }^{52}$

\section{$50 \quad$ Ibid}

51 Perla, A. (2007). El enfoque teórico de la cooperación internacional en educación. Análisis de tres agencias de cooperación internacional: IMEXCI, México; AECI, España y USAID, Estados Unidos (tesis de licenciatura en Relaciones Internacionales). Universidad de las Américas, Puebla, México.

52 Keohane, R. O. (1988). Después de la hegemonía: Cooperación y discordia en la política económica mundial. Grupo editor latinoamericano, p. 74. 
Por medio de esta definición se puede entender que la cooperación internacional está enfocada al desarrollo económico y social de los países que la realizan mediante la coordinación de políticas.

\section{B. La cooperación internacional en el derecho internacional}

La cooperación internacional encuentra su razón de ser en los principios universales de solidaridad entre los pueblos, respeto y protección de los derechos humanos y en la búsqueda incesante de mejores condiciones y mayores recursos que brinden al hombre una situación de bienestar conforme a su dignidad humana. Es por esto que en el derecho internacional entra a ser "una disciplina jurídica que regula las relaciones entre los Estados y entre estos y los organismos internacionales". 53

Con la creación de la ONU se buscó cumplir con el propósito de mantener la paz y la seguridad internacional, fomentar entre las naciones relaciones de amistad y realizar la cooperación internacional en la solución de problemas internacionales de carácter económico, social, cultural o humanitario, y en el respeto a los derechos humanos y libertades fundamentales. En la Declaración sobre los principios de derecho internacional referentes a las relaciones de amistad y la cooperación entre los Estados, de conformi-

53 La cooperación internacional y su régimen jurídico en Colombia (2007). Bogotá: Acción Social, p. 13. Recuperado de https://www. google.com.co/search?q=una+disciplina+jur\%C3\%ADdica+que+regu la+las+relaciones+entre+los+Estados+y+entre+estos+y+los+organis mos+internacionales\&oq=una+disciplina+jur\%C3\%ADdica+que+regu la+las+relaciones+entre+los+Estados+y+entre+estos+y+los+organis mos+internacionales\&aqs=chrome. .69i57.343j0j9\&sourceid=chrome \&espv=210\&es_sm=91\&ie=UTF-8 dad con la Carta de las Naciones Unidas de la Asamblea General, se proclama el principio de "La obligación de los Estados a cooperar entre sí, de conformidad con la Carta", en las diferentes esferas y en especial cooperar para promover el crecimiento económico en todo el mundo, particularmente en los países en desarrollo.

\section{Las convenciones de Viena}

Las convenciones de Viena sobre el derecho de los tratados entre Estados de 1969 y la Convención de Viena de los Tratados y Organizaciones Internacionales de 1986 son "codificaciones de prácticas consuetudinarias en el campo de los tratados internacionales", 54 y buscan reconocer la importancia cada vez mayor de los tratados como fuente del derecho internacional y como medio para desarrollar la cooperación pacífica entre las naciones. ${ }^{55}$ Esto se debe a que todas las normas y obligaciones internacionales deben ser cumplidas de buena fe por los sujetos a los que resultan oponibles, ${ }^{56} \mathrm{y}$ en dichas convenciones se encuentra plasmado, en el artículo 26 , bajo la rúbrica de pacta sunt servanda.

En la Convención de 1969, el artículo 27 establece que un Estado "no podrá invocar las disposiciones de su derecho interno como justificación del incumplimiento de un tratado", lo cual implica que cada tratado que entra en vigor

54 Ibid., p. 33.

55 Preámbulo de la Convención de Viena de 1969.

56 Ibid., p. 42. Hoy en día, este es un principio fundamental universalmente reconocido y enunciado por textos tan significativos como la Carta de las Naciones Unidas y la Declaración sobre los principios referentes a las relaciones de amistad y cooperación entre los Estados. 
obliga a las partes y debe ser cumplido por ella de buena fe. Además, el principio fundamental del derecho internacional pacta sunt servanda constituye la base esencial del derecho de los tratados, y “del funcionamiento armónico y pacífico de la comunidad internacional". 57

La Convención de Viena de 1988 sobre los Derechos de los Tratados se centra más en aclarar que las organizaciones internacionales son sujetos de derecho internacional, y que aunque nazcan por acuerdos entre Estados, “adquieren una subjetividad internacional propia diversa de aquella de los Estados que le dieron nacimiento, por lo cual su voluntad es autónoma y jurídicamente distinta de la de los Estados que la integran." 58 Debido a esto, el convenio también facilita la cooperación internacional ya que al tener estas "una naturaleza jurídica diversa de la de los Estados, con capacidad y reglas de organización especiales, crea un nuevo instrumento público, por medio del cual se codifica el derecho de los Tratados en relación con las organizaciones internacionales". ${ }^{59}$

Debido a esto, aunque no se haya llegado a ratificar un tratado, el conocido término de la norma ius cogens, que se encuentra establecido en el artículo 53 de la Convención de Viena de 1969, es definido como "una norma aceptada y reconocida por la comunidad internacional de Estados en su conjunto como norma que no ad-

57 Ibid.

58 Ibid.

59 Ibid., p. 34. mite acuerdo en contrario y que solo puede ser modificada por una norma ulterior de derecho internacional general que tenga el mismo carácter". Estas normas tienen que cumplir con los siguientes elementos: 1) Debe ser aceptada por la comunidad internacional de Estados en su conjunto; 2) Es una norma que no acepta norma en contrario, y 3) Solo puede ser modificada por otra norma ulterior de derecho internacional general que tenga el mismo carácter. ${ }^{60}$ Que sea aceptada por la comunidad internacional no implica que todos los Estados tengan que aceptarla, sino que, como establece la Comisión de Derecho Internacional (CDI) de la ONU, se requieren únicamente "todos los componentes esenciales de la comunidad internacional". ${ }^{61}$ Aunque no existe una respuesta tangente de cuáles son los "componentes esenciales", doctrinantes como Manuel Díez de Velasco han llegado a la conclusión de que se trata de normas que "pretenden dar respuesta a los intereses colectivos esenciales de todo el grupo social, que son propios de la nueva estructura comunitaria y que exigen reglas cualificadas por su grado de obligatoriedad, lo que conlleva la superior jerarquía de las mismas frente al resto de las normas del ordenamiento". 62

En este sentido, las normas consuetudinarias, como lo es la cooperación internacional, son

\footnotetext{
60 Abello Galvis, R. (2011). Introducción al estudio de las normas de ius cogens en el seno de la Comisión de Derecho Internacional, CDI. Biblioteca Jurídica Virtual del Instituto de Investigaciones Jurídicas de la UNAM, (123), 75-104, p. 90. Recuperado de http://www.juridicas. unam.mx/publica/librev/rev/vniver/cont/123/cnt/cnt4.pdf

$61 \quad$ Ibid

$62 \quad$ Ibid
} 
“normas que resultan de una práctica general aceptada como derecho, cuya existencia es independiente del derecho convencional".

\section{La cooperación internacional en el derecho del espacio ultraterrestre}

La cooperación internacional se considera "relacionada con las medidas de fomento de la confianza en el espacio ultraterrestre", ${ }^{63}$ y actualmente se encuentran tres clases de mecanismos internacionales en esta materia: mundiales, regionales y bilaterales.

Los Estados Partes de la ONU se han preocupado generalmente por dos esferas de la actividad espacial: la utilización del espacio ultraterrestre con fines pacíficos y la prevención de una carrera de armamento en el espacio ultraterrestre, por lo que, a través de la Comisión sobre la Utilización del Espacio Ultraterrestre y sus subcomisiones se dio lugar a la elaboración de cinco instrumentos internacionales que recogen los principios generales que deben regir la exploración y utilización del espacio ultraterrestre: el salvamento y la devolución de astronautas y la restitución de objetos lanzados al espacio ultraterrestre, la responsabilidad por daños causados por objetos espaciales, el registro de objetos lanzados al espacio ultraterrestre y las actividades en la Luna y otros cuerpos celestes. ${ }^{64}$ Además de una serie de actividades que ha organizado la ONU para ocuparse de la utilización del espacio ultraterrestre, se ha tomado

63 Asamblea General Naciones Unidas, op. cit., p. 64.

64 Ibid. en consideración la preocupación y el deseo de la comunidad internacional para prevenir una carrera de armamentos en el espacio ultraterrestre. Por esto, la Asamblea General redactó en su Documento Final de 1978 dicha preocupación y señaló que

para evitar la carrera de armamentos en el espacio ultraterrestre, deberían adoptarse nuevas medidas y celebrarse negociaciones internacionales apropiadas en consonancia con el espíritu del Tratado sobre los principios que deben regir las actividades de los Estados en la exploración y utilización del espacio ultraterrestre, incluso la Luna y otros cuerpos celestes. ${ }^{65}$

Además de los instrumentos internacionales creados por la ONU, existen otros que se ocupan de regular las actividades de los Estados de determinadas regiones en el espacio ultraterrestre. Esto se puede visualizar en diferentes organizaciones, tales como la Organización Internacional de Telecomunicaciones Espaciales (INTERSPUTNIK), ${ }^{66}$ la cual se creó con el fin de "satisfacer la demanda de algunos países de comunicaciones por teléfono y telégrafo y el intercambio de programas de radio y televisión, así como la transmisión de otros tipos de información vía satélite con miras a promover la cooperación política, económica y cultural". ${ }^{67}$

Otro estilo de organización fue creada en 1967 como un programa de cooperación general en-

65 Ibid., p. 65.

66 Esta organización fue creada en 1971 en virtud del acuerdo firmado en noviembre de 1971, y entró en vigor en 1972.

67 Ibid., p. 68. 
tre los países socialistas para la utilización con fines pacíficos del espacio ultraterrestre, denominado el Consejo de Cooperación Internacional en el Estudio y la Utilización del Espacio UItraterrestre (Intercosmos). ${ }^{68}$

Mecanismos bilaterales, como las negociaciones entre las potenciales mundiales (Ios Estados Unidos y la Unión Soviética) han sido muy exitosas. Entre ellos, el tratado sobre misiles antibalísticos de 1972 que prevé una Comisión Consultiva Permanente. Esta Comisión "ha sido el vehículo para la cooperación entre los Estados Unidos y la Unión Soviética en el fomento y aplicación de acuerdos firmados en el marco del SALT I y el SATL II". ${ }^{69}$ El más reciente, el Acuerdo Espacial entre los Estados Unidos y la Federación de Rusia de 1992 "sobre cooperación entre ambos países, brinda un amplio marco para la cooperación en relación con las actividades espaciales". ${ }^{70}$

\section{NORMATIVIDAD DEL DERECHO DEL ESPACIO ULTRATERRESTRE APROBADA POR CONSENSO}

\section{A. La Declaración de los principios jurídicos que deben regir las actividades de los Estados en la exploración $y$ utilización del espacio ultraterrestre}

La Declaración del 63, como es mejor conocida, fue aprobada por consenso por la Asamblea

\footnotetext{
68 Ibid.

69 Ibid., p. 70

$70 \quad$ Ibid
}

General en su Resolución 1962 (XVIII) del 13 de diciembre de 1963. Esta "contenía una serie de principios generales que caracterizaban el estatuto jurídico del espacio ultraterrestre y los cuerpos celestes y establecía el ámbito de la legalidad de las actividades de los Estado". ${ }^{71}$ Como era una resolución de la Asamblea General, se entendía que esta "no podía establecer normas vinculantes de derecho internacional. Sin embargo, ya durante el período de su aprobación se consideró que constituía la base de un futuro tratado jurídicamente vinculante". ${ }^{72}$

\section{B. Tratado sobre los principios que deben regir las actividades de los Estados en la exploración y utilización del espacio ultraterrestre, incluso la Luna y otros cuerpos celestes}

El tratado que se va a estudiar a continuación es usualmente conocido como el Tratado sobre el Espacio Ultraterrestre o como la Constitución del Espacio, y es uno de los más importantes ya que estableció las bases de la regulación internacional de las actividades espaciales. En el preámbulo del Tratado se establece el deseo de "contribuir a una amplia cooperación internacional en lo que se refiere a los aspectos científicos y jurídicos de la exploración y utilización del espacio ultraterrestre con fines pacíficos", con la esperanza de "que tal cooperación contribuirá al desarrollo de la comprensión mutua

71 kopal, V. (2009). Tratado sobre los principios que deben regir las actividades de los Estados en la exploración y utilización del espacio ultraterrestre, incluso la Luna y otros cuerpos celestes. United Nations Audiovisual Library of International Law, p. 2 
y al afianzamiento de las relaciones amistosas entre los Estados y los pueblos".

En los primeros tres artículos se puede establecer que por medio del tratado se reconoce el interés común que tienen todos los Estados en la explotación y utilización del espacio ultraterrestre, incluso la Luna y otros cuerpos celestes, como una zona "común" para todos los países sin importar el grado de desarrollo tecnológico, económico y científico, dado que esa exploración y explotación es del interés de toda la humanidad. Además, existe libre acceso a todas las zonas de los cuerpos celestes y están abiertos a la exploración y utilización en condiciones de igualdad, y de conformidad con el derecho internacional. Sin olvidar que se reconoce que el espacio ultraterrestre y todos los cuerpos celestes están abiertos a investigaciones científicas y que se promueve la cooperación internacional en estas; que se renuncia a la apropiación nacional por parte de cualquier Estado, y que se acepta la aplicación del derecho internacional en interés del mantenimiento de la paz y la seguridad internacional y del fomento de la cooperación internacional. ${ }^{73}$

\section{Acuerdo sobre el salvamento y la devolución de astronautas y la restitución de objetos lanzados al espacio ultraterrestre}

Dicho instrumento internacional tomó forma por medio de la Resolución 2345 (XXII) del 19 de diciembre de 1967, y entró en vigencia al siguiente año.
Este tratado se basa principalmente en el artículo $\mathrm{V}$ del tratado mencionado anteriormente, y lo que busca es establecer que aunque no se puede proclamar la soberanía del espacio ultraterrestre y de los cuerpos celestes, el Estado que haya registrado el lanzamiento de un objeto al espacio ultraterrestre tiene la jurisdicción y control sobre ese mismo y todo su personal durante toda la operación. ${ }^{74}$

Las obligaciones no solo se encuentran limitadas a los países que registran el lanzamiento de los objetos. Además de ellos, los demás países deben asistir en caso de un accidente, peligro o aterrizaje de emergencia a los astronautas, sin importar la nacionalidad de estos. Los países que asisten deben avisar al Estado de lanzamiento y a la Secretaría General de las Naciones Unidas sobre la situación, y utilizar todas las medidas de auxilio necesarias para prestarle ayuda a la tripulación del objeto en cuestión. Esto se debe a que los astronautas son considerados como enviados de la humanidad, y por lo tanto no importa a qué área geográfica de la Tierra entren. Ningún Estado tiene el derecho de alegar su soberanía y retenerlos por ingresar ilegalmente, sino la de atenderlos apenas aterricen y ser devueltos inmediatamente, sin demora, al Estado de registro del vehículo espacial.

Adicionalmente, este acuerdo establece que "en caso de que en el territorio de un Estado Parte se descubra un objeto espacial peligroso o piezas que lo compongan, la autoridad que

74 Kopal, V. y Diederiks-Verschoor, I. H. (2008). An Indroduction to Space Law. Estados Unidos: Wolters Kluwer Law \& Business, p. 32. 
haya lanzado el objeto tomará las medidas eficaces para eliminar las posibilidades de peligro o daño". ${ }^{75}$

Este tratado, animado por los sentimientos de humanidad, busca fomentar, a través del consenso, la cooperación internacional en la exploración y utilización del espacio ultraterrestre con fines pacíficos.

\section{Convenio sobre la responsabilidad internacional por daños causados por objetos espaciales de 1972}

El preámbulo del tratado en cuestión implícitamente establece que este fue redactado por los Estados, "convencidos de que el establecimiento de esas normas y procedimientos contribuirá a reforzar la cooperación internacional en el terreno de la exploración y utilización del espacio ultraterrestre con fines pacíficos".

Dicho tratado fue fácil de aprobar por medio de consenso debido a que existía "la necesidad de proteger a los terceros y asegurarles el pago rápido de la indemnización - plena y equitativaque merezcan por los daños que determinada actividad espacial les haga sufrir [...] [y] cuando Ios daños afecten a un sujeto que está realizando una actividad espacial, es decir, a un objeto espacial, a los bienes a bordo del mismo o a su tripulación". ${ }^{76}$ Además, con la creación del artículo VII del Tratado del 67 se establecieron

75 Recuperado de http://www.anepe.cl/2013/10/el-derecho-espacial/

76 Morales, V. y Manrique, A. M. (2012). Responsabilidad internacional por daños causados por objetos espaciales. Revista de Derecho Comunicaciones y Nuevas tecnologías, (7), 32.
“Ios elementos básicos sobre los cuales se desarrolló la responsabilidad por daños causados por objetos espaciales". Sin embargo, se dejaron por fuera del Tratado del 67 temas claves en materia de responsabilidad, como: "la delimitación o no de su concreción económica, la existencia y número de causas exonerantes de la responsabilidad, la normativa aplicable al cálculo de la indemnización o como el procedimiento de solución de las eventuales diferencias". ${ }^{77}$ Debido a esto, se hizo necesaria la creación de una norma internacional que estudiara todos los aspectos tomados en el Tratado del 67, y que aún no habían sido evaluadas.

\section{E. Convenio sobre el registro de objetos lanzados al espacio ultraterrestre de}

1975

Este tratado fue adoptado por medio de consenso el 12 de noviembre de 1974 y empezó a regir en 1977. Además de ser aprobado por Estados miembros, dos organizaciones internacionales (ESA y EUMESTAT) han declarado que también aceptan y se comprometen a los derechos y obligaciones entablados en él. ${ }^{78}$

Este tratado es de suma importancia, porque a través del registro del objeto espacial se contribuye a la preservación de la exploración y la explotación del espacio ultraterrestre para fines pacíficos debido a dos funciones esenciales que tiene. Primero que todo, sin un sistema de regis-

\footnotetext{
77 Gyula, G. (2012). En: Morales, V. y Manrique A. M. Responsabilidad internacional por daños causados por objetos espaciales. Revista de Derecho Comunicaciones y Nuevas tecnologías, (7), 5.

Kopal, op. cit., p. 44
} 
tro no es posible llevar el control de un vehículo espacial que ha causado daño; y segundo, a través de un sistema informativo y organizado de registro se puede minimizar la probabilidad y la desconfianza de armas de destrucción masiva siendo colocadas furtivamente en la órbita. ${ }^{79}$

Estos beneficios se encuentran plasmados en el preámbulo del tratado en cuestión, cuando establece que "un sistema obligatorio de registro de los objetos lanzados al espacio ultraterrestre ayudaría, en especial, a su identificación y contribuiría a la aplicación y el desarrollo del derecho internacional que rige la exploración y utilización del espacio ultraterrestre".

En el pasado se dejaba a la discreción de los Estados miembros registrar, por medio de la Secretaría General de la ONU ante el Copuos, la información del vehículo espacial que se iba a lanzar, pero no se volvió obligatorio sino solo hasta la entrada en vigencia de dicho tratado. ${ }^{80}$ Aunque era eficiente la labor realizada por la Secretaría General, la voluntariedad de la información hacía que esta fuera entregada tardíamente, por lo que no se ofrecía “mayor garantía para los países potencialmente víctimas de daños causados provocados por naves espaciales". ${ }^{81}$ Esto se establece con los artículos II y III, ya que se crea en esencia la obligación de los Estados miembros de mantener "un registro apropiado"

79 Ibid.

80 Ibid.

81 Irigoin, J. (2013). El derecho espacial. Anepe-Ministerio de Defensa Nacional. Recuperado de http://www.anepe.cl/2013/10/el-derechoespacial/ y de la Secretaría General de la ONU de mantener un registro de acceso pleno y libre.

El artículo IV del tratado establece los requerimientos necesarios para el registro, y entre esos se exige que se dé una descripción de la función general del objeto espacial. Este sigue siendo un problema para los Estados dado que se evidencia la falta de colaboración para revelar que han lanzado satélites con propósitos militares o cualquiera que sea la real misión. La razón para esto es que todavía existe un alto grado de desconfianza entre los Estados. ${ }^{82}$ Por otro lado, no podemos olvidar la existencia de la Resolución 62/101 del 17 de diciembre de 2007 , en la cual se complementan y motivan una seria de nuevos elementos a registrarse debido al desarrollo constante de nuevas tecnologías, y al aumento de números de Estados que realizan actividades espaciales, al igual que el de la cooperación internacional y de las actividades espaciales Ilevadas a cabo por entidades no gubernamentales, así como asociaciones constituidas por entidades no gubernamentales de más de un país.

\section{F. Acuerdo que debe regir las actividades de los Estados en la Luna y otros cuerpos celestes de 1979}

Este tratado ha sido mejor conocido como el Tratado de la Luna. En el preámbulo del mismo se establece "que la Luna, como satélite natural de la Tierra, desempeña un papel importante en la exploración del espacio ultraterrestre". Por lo

82 Kopal, op. cit., p. 45. 
tanto promueve la igualdad y el arduo trabajo necesario en el desarrollo de la cooperación entre los Estados en la exploración y explotación de la Luna y de los otros cuerpos celestes, dado que será en beneficio del pueblo, y al mismo tiempo "deseando evitar que la Luna se convierta en zona de conflictos internacionales".

Ha habido consenso en cuanto a que los Estados partes están de acuerdo en que la Luna, sus recursos naturales y otros cuerpos celestes "son patrimonio común de la humanidad y que [...] se comprometen a establecer un régimen internacional apropiado que regule la explotación de los recursos naturales de la Luna, cuando esa explotación sea viable". 83

Teniendo en cuenta esto, el artículo 9 del tratado busca dejar claro que la Luna no debería ser objeto de apropiación nacional al establecer que "las estaciones deberán estar dispuestas de modo que no entorpezcan el libre acceso a todas las zonas de la Luna del personal, los vehículos y el equipo de otros Estados partes que desarrollan actividades en la Luna". Adicionalmente deja claro, por medio de su artículo 6, que todos los Estados partes tienen el derecho a recoger y extraer de la Luna muestras de sus minerales y otras substancias y estos quedarían a la disposición de los demás países y de la comunidad científica internacional.

El consenso se establece en este caso para dejar claro que los intereses y las necesidades de los países en desarrollo y aquellos que aporta-

\footnotetext{
83 Irigoin, op. cit.
}

ron directa o indirectamente a la exploración de la Luna son tomados en especial consideración.

\section{OBLIGATORIO CUMPLIMIENTO DE LAS NORMAS DEL DERECHO DEL ESPACIO ULTRATERRESTRE}

En el momento en la historia en que empezó el desarrollo tecnológico dirigido a explorar y explotar el espacio ultraterrestre, la comunidad internacional no se encontraba preparada para eso. No existía alguna experiencia de lo que fuese una actividad espacial, por lo que surgió una gran preocupación y se empezaron a crear organismos dedicados a estudiarlos. Con la creación de la Resolución del 63, que dio pie a la Declaración de los principios jurídicos que deben regir las actividades espaciales, la cual fue base para el Tratado del 67, se establecieron principios innegociables para que el derecho del espacio ultraterrestre pudiese ser un espacio en el que se podría desarrollar una tecnología que funcionara a favor del hombre y no en su contra.

Hasta el día de hoy no todos los países han llegado a ratificar los tratados del derecho del espacio ultraterrestre, dado que no han llevado a cabo el proceso necesario para hacerlo por medio de la legislación interna de cada Estado. Tratados como el Tratado de la Luna solo han sido ratificados por seis Estados partes. Sin embargo, esto no implica que solo vaya a regir a estos Estados. Todos los demás países se encuentran obligados por el principio de ius cogens, ${ }^{84}$ que

84 Ius cogens es derecho coercitivo, obligatorio, que se utiliza para designar una norma imperativa en el derecho internacional. 
protegen valores esenciales compartidos por la comunidad internacional.

El artículo 53 de la Convención de Viena sobre Derecho de los Tratados establece que estas no pueden ser derogadas, ni por normas internas de los Estados ni por normas diferentes. Solo pueden ser derogadas por aquellas que tengan el mismo rango, ya que cualquier tratado internacional contrario a una norma ius cogens se entendería como nula. Los principios establecidos en la redacción del Tratado del 67 se pueden entender como normas imperativas pues "el espacio es patrimonio común de la humanidad" .85

Adicionalmente, es pertinente establecer que el Tratado del 67 encuentra su fundamento en la Carta de las Naciones Unidas, y si este no ha sido ratificado por todos los Estados miembros no implica que no rija para todos los Estados. Esto se debe a que la Carta de las Naciones Unidas ha sido firmada y ratificada por todos los Estados. La Carta, en su preámbulo, establece que "las normas del derecho internacional consuetudinario han de continuar rigiendo las cuestiones que no hayan sido expresamente reguladas en las disposiciones de la presente Convención". Por ende, de acuerdo con lo establecido en la Convención recientemente citada en su artículo 38, se entiende que la costumbre, que es fuente de derecho, actúa como norma imperativa y por lo tanto es ius cogens, dado que la costumbre en el plano internacional y en el derecho

85 Rodríguez, E. Nuestro derecho al espacio. la órbita geoestacionaria: ¿una frustrada regulación? Biblioteca Jurídica Virtual del Instituto de Investigaciones Jurídicas de la UNAM. Revista de Temas Constitucionales, p. 56. Recuperado de http://www.juridicas.unam.mx/publica/ librev/rev/juicio/cont/2/cnt/cnt4.pdf del espacio ultraterrestre goza de una fuerza coercitiva, inclusive mayor que las normas positivas. Por lo tanto, los principios acogidos en el Tratado del 67 y demás declaraciones y resoluciones tienen que ser acogidos por todos Ios Estados, inclusive sin haber sido ratificados, debido a que son considerados como derecho consuetudinario, y dicho incumplimiento tendría consecuencias en el campo jurídico.

En el derecho del espacio ultraterrestre, se podría establecer, al no actuar los Estados en contra de un tratado, resolución o principio existente, se acepta lo que en estos se busca regular. Esto es visible en el caso colombiano, por cuanto no hemos ratificado el Tratado del 67. Sin embargo, no hemos llevado a cabo ningún tipo de actuación que vaya en contra del principio de no apropiación, por lo que se entiende que aceptamos y cumplimos lo establecido en él.

\section{CONCLUSIONES}

En el derecho del espacio ultraterrestre es importante fundamentarse en el consenso, en la confianza y en la cooperación internacional. Se puede afirmar que el principio de la cooperación, la confianza y el consenso son sus pilares fundamentales. Esto se debe a que algunas de las normas que regulan la exploración, explotación y utilización del espacio ultraterrestre (como los principios, tratados y resoluciones que existen y que se encuentran vigentes) aún no han sido ratificadas por todos los Estados. Sin embargo, pensamos que siguen siendo de obligatorio cumplimiento, puesto que de la Carta de las $\mathrm{Na-}$ 
ciones Unidas hacen parte la costumbre, que es reconocida como norma ius cogens, y esta sí ha sido ratificada por todos los Estados miembros. Adicionalmente, todas las normas del derecho del espacio ultraterrestre han sido aprobadas por consenso, lo cual no significa que todos hayan aprobado o votado a favor, sino más bien que no se ha manifestado oposición en contra de lo establecido. En otras palabras, que no va a darse una actuación en contra sin importar que no se esté de acuerdo con lo establecido.

En el desarrollo del derecho del espacio ultraterrestre, ningún Estado ha presentado oposición persistente a ninguna de las normas, por lo que debe entenderse que al nacer una norma por medio del consenso, no hay disenso, que es lo contrario. Es decir, se acepta lo que las otras partes han planteado. Al no oponerse a lo que los demás están diciendo, se está generando confianza en que se va a cumplir con lo acordado. Al generar confianza, los Estados están dispuestos a cooperar unos con otros, y de esta manera será más probable desarrollar tecnología en el derecho del espacio ultraterrestre, dado que los países estarán más dispuestos a apoyarse mutuamente para lograr nuevos avances en el terreno del espacio ultraterrestre.

Se hizo necesario buscar una nueva fuente de origen del derecho internacional, en el cual todo lo que se pactara fuera por medio del consenso. Por eso, el consenso es la fuente del derecho internacional. No quiere decir que los tratados no sean fuente, sino que todos estos tratados fueron consensuados. El derecho espacial, debido a su naturaleza, es un campo en el que surgieron una serie de formas en las cuales las normas se sienten obligatorias aunque no han sido pactadas por medio de tratados, sino por acuerdos consensuados entre los Estados. Es este consenso el que le da nacimiento al derecho del espacio ultraterrestre, dado que por medio de este los Estados se obligan.

\section{BIBLIOGRAFÍA}

\section{Doctrina}

Keohane, R. O. (1988). Después de la hegemonía. Cooperación y discordia en la política económica mundial. Grupo editor latinoamericano.

Kopal, V. (2009). Tratado sobre los principios que deben regir las actividades de los Estados en la exploración y utilización del espacio ultraterrestre, incluso la Luna y otros cuerpos celestes. United Nations Audiovisual Library of International Law.

kopal V. y Diederiks-Verschoor, D. I. H. (2008). An Indroduction to Space Law. Estados Unidos: Wolters Kluwer Law \& Business.

Ospina Fernández, G. (1998). Teoría general del contrato y del negocio jurídico (5. ${ }^{a}$ ed.). Bogotá: Temis.

Valencia Zea, A. (1981). Derecho civil. Parte general y personas, t. I (9. a ed.). Bogotá: Temis. 
Documentos de las Naciones Unidas, tratados y convenios

Acuerdo que debe regir las actividades de los Estados en la Luna y otros cuerpos celestes

APÉNDICE II. Directrices sobre tipos apropiados de medidas de fomento de la confianza y sobre la aplicación de tales medidas en los planos mundial o regional. Asamblea General de las Naciones Unidas, Resolución 39/63 del 12 de diciembre de 1984

Carta de las Naciones Unidas

Convención de Viena sobre el Derecho de los Tratados de 1969

Convención sobre la Responsabilidad Internacional por Daños Causados por Objetos Espaciales

Tratado sobre los principios que deben regir las actividades de los Estados en la exploración y utilización del espacio ultraterrestre, incluso la Luna y otros cuerpos celestes (Tratado de 1967)

\section{Artículos}

Abello Galvis, R. Introducción al estudio de las normas de ius cogens en el seno de la comisión de Derecho Internacional, CDI. Biblioteca Jurídica Virtual del Instituto de Investigaciones Jurídicas de la UNAM, (123), 75-104, p. 90. Recuperado de http://www.juridicas.unam.mx/publica/librev/rev/vniver/ cont/123/cnt/cnt4.pdf
Morales, V. y Manrique, A. M. (2012). Responsabilidad internacional por daños causados por objetos espaciales. Revista de Derecho Comunicaciones y Nuevas tecnologías (7).

Onghena, Y. (2003). ¿Por qué la confianza? Revista CIDOB d'Afers Internacionals, (61-62).

Perla, A. (2007). El enfoque teórico de la cooperación internacional en educación. Análisis de tres agencias de cooperación internacional: IMEXCI, México; AECI, España y USAID, Estados Unidos (tesis de licenciatura en Relaciones Internacionales). Universidad de las Américas, Puebla, México.

Varela, V. (2012). La importancia de fomentar la cooperación internacional en la utilización y exploración del espacio ultraterrestre (tesis de pregrado). Universidad Javeriana de Cali, Cali, Colombia.

\section{Páginas webs}

Acción Social (2007). La cooperación internacional y su régimen jurídico en Colombia, Bogotá, p. 13. Recuperado de https://www. google.com.co/search?q=una+disciplina + j ur\%C3\%ADdica+que+regula+las+relacione $\mathrm{s}+$ entre+los+Estados+y+entre+estos+y+los +organismos+internacionales\&oq=una+dis ciplina+jur\%C3\%ADdica+que+regula+las+r elaciones+entre+los+Estados+y+entre+est os+y+los+organismos+internacionales\&aqs =chrome..69i57.343j0j9\&sourceid=chrome \&espv=210\&es_sm=91\&ie=UTF-8 
Ansuátegui, F. J. Consenso y derecho, p. 59. Recuperado de http://e-archivo.uc3m.es/bitstream/handle/10016/9399/consenso_ansuategui_2008.pdf;jsessionid=39DF33AAC5 78729435C6COBC492DE506?sequence $=1$

Briggs, B. Introducción al proceso de consenso. Instituto Internacional de Facilitación y Consenso, IIFAC, p. 6. Recuperado de http://proyectocultivandovida.pbworks.com/f/Introduc cion+al+proceso + de+consenso.pdf

Definición de “consenso". Definición.De. Recuperado de http://definicion.de/consenso/

Fukuyama, F. La gran ruptura. Hevia de la Jara, F. ¿Cómo construir confianza? Hacia una definición relacional de la confianza social. Instituto de Investigaciones Jurídicas UNAM, p. 18. Recuperado de http://www.redacademica.edu.co/archivos/redacademica/proyectos/ddhh/autoformacion_ddhh/unidad10/ anexo_10-23_como_construir_confianza.pdf

Gutiérrez, C. La crisis del derecho del espacio. Un desafío para el derecho internacional del nuevo siglo, p. 249. Recuperado de http://dspace.unav.es/dspace/bitstream/10171/22202/1/ADI_XV_1999_06. pdf

Hevia de la Jara, F. ¿Cómo construir confianza? Hacia una definición relacional de la confianza social. Instituto de Investigaciones Jurídicas UNAM, p. 18. Recuperado de http://www. redacademica.edu.co/archivos/redacademica/proyectos/ddhh/autoformacion_ddhh/ unidad10/anexo_10-23_como_construir_ confianza.pdf

Irigoin, J. El derecho espacial. Anepe-Ministerio de Defensa Nacional. Recuperado de http:// www.anepe.cl/2013/10/el-derecho-espacial/

NASA. Sputnik and the dawn of the spage age. The space race. Recuperado de http://history.nasa.gov/sputnik/

Principios que rigen a los tratados internacionales de derecho público. Derecho Constitucional. Universidad Sergio Arboleda. Recuperado de ehttp://www.usergioarboleda.edu.co/ derecho/derecho_constitucional/articulos_ opinion_analisis_principiosquerigenlostratadosinternacionales.htm

Ortiz, E. y Manuel, J. Consenso y disenso. Recuperado de www.scribd.com/doc/83020100/ Consenso-y-Disenso

Rodríguez, E. Nuestro derecho al espacio. La órbita geoestacionaria: ¿una frustrada regulación? Biblioteca Jurídica Virtual del Instituto de Investigaciones Jurídicas de la UNAM. Revista de Temas Constitucionales, p. 56. Recuperado de http://www.juridicas.unam.mx/publica/librev/rev/juicio/cont/2/cnt/cnt4.pdf

Valero, J. Sociedades de cultura versus sociedades de desconfianza. Universidad de Valladolid, p. 213. Recuperado de http://www. google.com/url?sa=t\&rct=j\&q=\&esrc=s\&sou rce $=$ web \&cd $=1 \& v e d=0 C C g Q F j A A \& u r l=h t t p \%$ 
3A\%2F\%2Fdialnet.unirioja.es\%2Fdescarga\% 2Farticulo\%2F1138368.pdf\&ei=TmOEUs7o H9CLkAeV54D4AQ\&usg=AFQjCNG2wkMOxA xJkNmM3sPwlqn39e2_5A\&bvm=bv.563433 20,d.eWo
W. V. Rauchhaupt, H. El sistema del derecho espacial, p. 203. Recuperado de http:// www.ordenjuridico.gob.mx/Publicaciones/ CDs2010/CDEspacio/pdf/OP12.pdf 